\title{
Structure-Dependent Thermal Defunctionalization of Single-Walled Carbon Nanotubes
}

\author{
Saunab Ghosh, Fang Wei, Sergei M. Bachilo, Robert H. Hauge, W. E. Billups and R. Bruce Weisman* \\ Department of Chemistry and R.E. Smalley Institute for Nanoscale Science and Technology, \\ Rice University, 6100 Main Street, Houston, Texas 77005 USA
}

email address: weisman@rice.edu

\section{ABSTRACT}

Covalent sidewall functionalization of single-walled carbon nanotubes (SWCNTs) is an important tool for tailoring their properties for research purposes and applications. In this study, SWCNT samples were first functionalized by reductive alkylation using metallic lithium and 1iodododecane in liquid ammonia. Samples of the alkyl-functionalized SWCNTs were then pyrolyzed under an inert atmosphere at selected temperatures between 100 and $500{ }^{\circ} \mathrm{C}$ to remove the addends. The extent of defunctionalization was assessed using a combination of thermogravimetric analysis, Raman measurements of the RBM, D, and G bands, absorption spectroscopy of the first- and second-order van Hove peaks, and near-IR fluorescence spectroscopy of $(n, m)$-specific emission bands. These measurements all indicate a substantial dependence of defunctionalization rate on nanotube diameter, with larger diameter nanotubes showing more facile loss of addends. The effective activation energy for defunctionalization is estimated to be a factor of $\sim 1.44$ greater for $0.76 \mathrm{~nm}$ diameter nanotubes as compared to those with $1.24 \mathrm{~nm}$ diameter. The experimental findings also reveal the quantitative variation with functionalization density of the Raman D/G intensity ratio and the relative near-IR 
fluorescence intensity. Pyrolyzed samples show spectroscopic properties that are equivalent to those of SWCNTs prior to functionalization. The strong structure dependence of defunctionalization rate suggests an approach for scalable diameter-sorting of mixed SWCNT samples.

KEYWORDS: covalent functionalization; pyrolysis; dodecylation; D/G ratio; nanotube fluorescence restoration 
Single-walled carbon nanotubes (SWCNTs) are among the most intensely studied artificial nanomaterials because of their unusual fundamental properties and promise for diverse applications. An essential tool for tailoring the interactions of SWCNTs with their environment is functionalization. ${ }^{1}$ Such functionalization may be either noncovalent, for example with coatings of surfactants, synthetic polymers, or biopolymers ${ }^{2}$ or covalent, achieved through chemical reactions that link addends to sidewall carbon atoms of the nanotube. ${ }^{3-6}$ Both noncovalent and covalent functionalization are commonly used to allow SWCNTs to be individually suspended or dissolved in liquid media. In addition, one can sometimes exploit structure-specific interactions that depend on nanotube diameter and roll-up (chiral) angle. As an example, noncovalent interactions between nanotubes and the organic polymeric dye PFO are selective enough that it can extract a subset of SWCNT structures from broadly heterogeneous as-grown samples. ${ }^{7}$ Covalent sidewall functionalization offers another possible route to SWCNT sorting, based on structure-dependent reactivity patterns. ${ }^{8,9}$ The main such pattern is enhanced reactivity of smaller diameter nanotubes because of their greater $\mathrm{sp}^{2}$ bond strain. ${ }^{10}$ Although previously unexplored, variations in reactivity with structure should also affect the process of covalent defunctionalization, in which bonds to addends are broken to regenerate pristine SWCNTs.

We report here a structure-resolved study of thermally induced defunctionalization reactions. Pristine SWCNT samples were first subjected to Billups-Birch reductive alkylation to obtain substantial sidewall functionalization with docedyl groups and solubility in organic solvents. ${ }^{3,5}$ These dodecylated samples were then pyrolyzed for specific times at a range of controlled temperatures, after which changes in composition were monitored by optical absorption, fluorescence, and Raman spectroscopies and gravimetric analysis. Our results reveal that the thermal defunctionalization depends strongly on nanotube diameter, suggesting a new experimental tool for achieving scalable sorting of SWCNT mixtures. 


\section{RESULTS AND DISCUSSION}

Raman Spectroscopy. The Raman spectrum of the solid alkylated SWCNT sample, shown in the top trace of Figure 1a, reveals an intense D band near $1300 \mathrm{~cm}^{-1}$. As it is well known that the D-band can be induced by $\mathrm{sp}^{3}$ sites, ${ }^{11}$ this indicates extensive covalent functionalization of the nanotubes. To monitor thermal defunctionalization, portions of this dodecylated sample were pyrolyzed for 30 minutes at various temperatures. As can be seen from the other traces in Figure 1, the spectral width, peak frequency, and relative intensity of the D-band all decrease systematically as the pyrolysis temperature is increased. We interpret these changes as reflecting the loss of dodecyl addends to give more sparsely functionalized SWCNTs. A plot of the D/G band peak intensity ratio vs. pyrolysis temperature (Figure 1b) shows a particularly sharp drop between 150 and $200{ }^{\circ} \mathrm{C}$. Treatment at the highest pyrolysis temperature of $500{ }^{\circ} \mathrm{C}$ reduces the $\mathrm{D} / \mathrm{G}$ ratio to only 0.06 , a value typical of many SWCNT samples that have never undergone functionalization reactions. Pyrolysis also sharpens the G band, allowing a Raman feature near $1554 \mathrm{~cm}^{-1}$ to be resolved in samples defunctionalized at $180{ }^{\circ} \mathrm{C}$ and above. These observations are consistent with earlier reports on thermal detachment of fluorine atoms and alkyl addends covalently bound to SWCNT sidewalls. ${ }^{5,12}$

SWCNTs show distinct radial breathing mode (RBM) resonance Raman features at low frequencies. Each nanotube species gives a specific RBM peak whose frequency is inversely related to nanotube diameter and can often be used to deduce its $(n, m)$ indices. ${ }^{13}$ Figure 1c shows a set of RBM spectra measured with $633 \mathrm{~nm}$ excitation on solid dodecylated SWCNT samples that had been pyrolyzed for $30 \mathrm{~min}$ at different fixed temperatures. Before pyrolysis, the heavily functionalized sample did not exhibit any sharp RBM bands, likely because strong disruption of the $\pi$-electron system had suppressed the resonance enhancement effect. However, after defunctionalization at $140{ }^{\circ} \mathrm{C}$, an $\mathrm{RBM}$ band appeared at $\sim 193 \mathrm{~cm}^{-1}$. This band is assigned to nanotubes with a diameter near $1.24 \mathrm{~nm}$, probably the metallic 
species $(9,9)$. Pyrolysis at the higher temperature of $200{ }^{\circ} \mathrm{C}$ induced additional RBM peaks attributed to $(12,3)$ and $(10,3)$ SWCNTs, which have diameters of 1.09 and $0.94 \mathrm{~nm}$ respectively. After $300{ }^{\circ} \mathrm{C}$ pyrolysis, RBM transitions of the $(7,5)(0.84 \mathrm{~nm})$ and $(8,3)(0.78 \mathrm{~nm})$ species also became evident. Another view of this pattern can be seen in Figure 1d, where we plot RBM intensities (relative to the feature near $\left.193 \mathrm{~cm}^{-1}\right)$ from $(7,5)$ and $(12,3)$ SWCNTs in a set of dodecylated samples pyrolyzed at closely spaced temperatures. The larger diameter $(12,3)$ nanotubes show maximum recovery of their RBM signals with pyrolysis at $350{ }^{\circ} \mathrm{C}$, whereas temperatures greater than $500{ }^{\circ} \mathrm{C}$ appear necessary for full restoration from the smaller diameter $(7,5)$ nanotubes. Additional data for the $(10,3)$ and $(8,3)$ species are included in Supporting Information. It seems clear that the pattern of recovery of the RBM Raman bands shows structure-dependent defunctionalization rates, with the largest diameter SWCNTs most readily losing their dodecyl addends.

To confirm these observations while avoiding possible sampling errors that may arise from local measurements on inhomogeneous solid samples, we acquired similar Raman spectra from dispersions of pyrolyzed dodecylated SWCNTs in aqueous surfactant solutions. The $671 \mathrm{~nm}$ excitation laser used in these experiments was nearly resonant with the $(8,3) \mathrm{E}_{22}$ transition, ${ }^{14,15}$ so the spectra are dominated by $(8,3)$ features. Samples were matched in absorbance to allow quantitative comparisons. Figures $2 \mathrm{a}$ and $2 \mathrm{~b}$ show that RBM and G-band intensities increased strongly with increasing pyrolysis temperature while the D-band diminished. Plots of the $(8,3) \mathrm{RBM}$ and G-band intensities vs. pyrolysis temperature (Figure 2c) reveal a much sharper temperature dependence for the RBM peak. This probably reflects the fact that the G-band contains superimposed contributions from multiple $(n, m)$ species that lie within the resonance excitation window but have different pyrolysis profiles, whereas the RBM feature is specific to $(8,3)$. In Figure $2 \mathrm{~d}$ we compare the RBM signals (normalized to their $500{ }^{\circ} \mathrm{C}$ values) $v s$. pyrolysis temperature for three SWCNT species ranging in diameter from 0.78 to $1.04 \mathrm{~nm}$. In agreement with our 
solid sample findings described above, we find that the onset temperature for significant defunctionalization increases with decreasing SWCNT diameter.

In order to more precisely identify these $(n, m)$-specific onset temperatures for defunctionalization by 30 min pyrolysis treatments, we used four different Raman excitation lasers to monitor the appearance of distinct RBM features. Each frame of Figure 3 shows species-selected Raman spectra on the same intensity scale for two closely spaced pyrolysis temperatures. The large relative increases in RBM intensities between the two traces allows threshold temperatures to be defined within $\sim 10{ }^{\circ} \mathrm{C}$ for the SWCNT species displayed here plus several others. These results are compiled in Table 1 and plotted as a function of SWCNT diameter in Figure 4, which shows a smooth, monotonic decrease in defunctionalization threshold temperature with nanotube diameter. Points for the two metallic and eleven semiconducting species fall on the same curve, suggesting that the observed dependence is independent of SWCNT metallicity.

If we assume that the kinetics of SWCNT thermal defunctionalization follows an Arrhenius form, then the data in Table 1 allow one to estimate relative activation energies. At their listed threshold temperatures, the $(n, m)$ species react to a similar extent during the 30 min pyrolysis, implying similar reaction rate constants $k=A \exp \left[-E_{a} / R T_{t h r}\right]$. If pre-factors $A$ are nearly equal for the different $(n, m)$ species, as seems plausible within this class of closely related reactions, then activation energies $E_{a}$ must be proportional to the experimental threshold temperatures (expressed in kelvin). The last column of Table 1 lists these deduced activation energies relative to that of the largest diameter SWCNTs studied here. This analysis finds that the defunctionalization activation energy is $44 \%$ higher for $(6,5)$ than for $(9,9)$ SWCNTs. Moreover, if the defunctionalization involves homolytic bond cleavage to radicals, then there is no significant energy barrier to the reverse reaction and these activation energies equal the addend binding energies. We attribute the observed variation in binding energies to increased reactivity 
of smaller diameter SWCNTs caused by curvature-induced strain and the greater release of that strain energy on $\mathrm{sp}^{3}$ functionalization. ${ }^{16}$ Of course, there may also be complicating factors that give a distribution of activation energies even for a single $(n, m)$ species, such as variations among nanotubes in the initial addend density and addition pattern, including banding, ${ }^{17-20}$ and resulting variations in binding energies due to cooperative effects. Our findings should therefore be viewed as averages over any such distributions. We also note that the prior experimental study of Liang et al. suggests that our samples underwent mainly 1,2-addition of dodecyl addends rather than the 1,4-addition thought to dominate when sodium is used as the reducing agent..$^{5}$

Thermogravimetric Analysis. We performed thermogravimetric analysis (TGA) on dodecylated samples to determine the extent of initial functionalization and to correlate weight loss data with the Raman pyrolysis results described above. Using continuous heating of dodecylated SWCNT samples under an argon atmosphere from 50 to $500{ }^{\circ} \mathrm{C}$ with a ramp of $2{ }^{\circ} \mathrm{C} \min ^{-1}$, we observed a mass loss of approximately 50\%. The data are plotted as the solid curve in Figure 5a. After accounting for the $22 \%$ residual iron catalyst content in our sample, we deduced that the mass loss during pyrolysis implied an initial composition of one $\mathrm{C}_{12} \mathrm{H}_{25}$ group per 11.2 SWCNT carbon atoms, or an average linear density of approximately $9 \mathrm{C}_{12} \mathrm{H}_{25}$ groups per nanometer along the nanotube axis. The dotted curve in Figure 5a shows the temperature derivative of the mass loss, with a peak near $160{ }^{\circ} \mathrm{C}$. This result is consistent with the data of Figure 1b, in which the sharpest drop in $\mathrm{I}_{\mathrm{D}} / \mathrm{I}_{\mathrm{G}}$ Raman ratio, which we interpret as defunctionalization, also occurs near $160^{\circ} \mathrm{C}$.

We made additional mass loss measurements on a set of 22 equivalent samples of dodecylated SWCNTs. In this experiment, each sample was pyrolyzed for 30 minutes at a specific fixed temperature before measurement of the final mass. The ratios of final to initial mass, expressed as percentages, are plotted vs. pyrolysis temperature in Figure 5b. These results are quite similar to those shown above in 
Figure 5a, despite differences in measurement methods. The right scale of Figure $5 \mathrm{~b}$ shows the numbers of $\mathrm{C}_{12} \mathrm{H}_{25}$ addends per nanotube carbon atom that correspond to the plotted mass percentages. By combining the results of Figures $5 \mathrm{~b}$ and $1 \mathrm{~b}$, we can quantitatively deduce how the Raman $\mathrm{I}_{\mathrm{D}} / \mathrm{I}_{\mathrm{G}}$ ratio depends on extent of dodecyl functionalization. As shown in Figure 6, this variation is sigmoidal in shape. The $\mathrm{I}_{\mathrm{D}} / \mathrm{I}_{\mathrm{G}}$ ratio increases nearly linearly at low addend densities, then curves upward until it passes an inflection point and appears to approach a maximum of approximately 1.1 near 0.1 addends per carbon atom. A previous study of diazonium-functionalized SWCNTs also found a nonlinear dependence of $\mathrm{I}_{\mathrm{D}} / \mathrm{I}_{\mathrm{G}}$ ratio on extent of functionalization, although in that case the ratio (determined using a Raman laser that probed metallic SWCNTs) went through a maximum near addend densities of only 0.01 per carbon atom. ${ }^{11,21} \mathrm{~A}$ recent report shows the integrated $\mathrm{D} / \mathrm{G}$ ratio increasing monotonically to values above 0.6 as a diazonium functionalization reaction proceeds. ${ }^{22}$ It is not yet clear whether the observed differences in $\mathrm{I}_{\mathrm{D}} / \mathrm{I}_{\mathrm{G}}$ functionalization curves reflect differences in SWCNT metallicity, addend identity, or perhaps other factors.

Absorption and Fluorescence Spectroscopy. Because SWCNT optical transitions can also be strongly affected by covalent sidewall functionalization, we studied the electronic absorption and emission spectra of our dodecylated samples following $30 \mathrm{~min}$ pyrolysis treatments at various temperatures. Figure 7a shows a set of aqueous suspension absorption spectra (vertically offset for clarity) for dodecylated SWCNTs before pyrolysis (trace labeled as $25^{\circ} \mathrm{C}$ ) and for dodecylated samples that had been pyrolyzed at temperatures from 200 to $500{ }^{\circ} \mathrm{C}$. The unpyrolyzed sample has a featureless absorption spectrum with no trace of the sharp van Hove peaks that are characteristic of SWCNTs with intact $\pi$-electron systems. Broad and indistinct van Hove features appear after $200{ }^{\circ} \mathrm{C}$ pyrolysis, and they become much sharper after treatment at $300{ }^{\circ} \mathrm{C}$ and above. Moreover, the $\mathrm{E}_{11}$ transitions near 950 $\mathrm{nm}$, which arise from the smallest diameter SWCNTs in the sample, are restored only at the highest 
pyrolysis temperatures. $\mathrm{E}_{22}$ absorptions (in the visible wavelength range) seem to be restored at somewhat lower pyrolysis temperatures than the $\mathrm{E}_{11}$ features.

Figure $7 \mathrm{~b}$ shows fluorescence spectra of these samples when excited at $659 \mathrm{~nm}$. The plotted intensities have been scaled by sample absorbance at $300 \mathrm{~nm}$ to compensate for concentration differences. The sample with fully functionalized nanotubes showed no detectable emission, as is consistent with disruption of the SWCNT $\pi$-electron system by numerous $\mathrm{sp}^{3}$ sites $^{23,24}$ After pyrolysis at $200{ }^{\circ} \mathrm{C}$, weak and broad emission appears from approximately 1150 to $1420 \mathrm{~nm}$. Pyrolysis at $240{ }^{\circ} \mathrm{C}$ intensifies this emission and extends it to $\sim 1050 \mathrm{~nm}$. Traces recorded after pyrolysis at 300, 400, and $500{ }^{\circ} \mathrm{C}$ show successively sharper and stronger emission. The peaks at shorter wavelengths, from smaller diameter SWCNTs such as $(8,3)$ and $(7,5)$, intensify at higher processing temperatures and blueshift more than those from larger diameter species. This is consistent with the pattern deduced from Raman and absorption spectra showing that defunctionalization is more facile for larger diameter SWCNTs. The data of Fig. 7b can be spectrally integrated and plotted versus pyrolysis temperature to obtain a coarse-grained view of overall sample defunctionalization. As shown in Fig. 7c, this plot reveals a 10-fold increase in fluorescence recovery over the pyrolysis range of 200 to $500{ }^{\circ} \mathrm{C}$ and suggests that some additional recovery might be obtained by more vigorous pyrolysis. Finally, the relation between pyrolysis temperature and average extent of functionalization from Fig. $5 \mathrm{~b}$ can be used to recast the $\mathrm{x}$-axis of Fig. 7c and obtain Fig. 7d, which shows that overall sample fluorescence decreases nearly linearly with increasing addend density. Extrapolation of the linear trend suggests that complete fluorescence quenching occurs at an average functionalization density of $\sim 0.05$ dodecyl groups per carbon atom. In the absence of addend banding or clustering, this would correspond to a typical spacing between addends of $\sim 5 \mathrm{~nm}$ along the nanotube axis. Because this spacing is much smaller than the mean exciton excursion ranges deduced from single-particle quenching experiments, ${ }^{24,25}$ it would 
suggest that dodecyl-functionalized $\mathrm{sp}^{3}$ sites on the SWCNT sidewall are less efficient exciton quenchers than the phenyl halide-functionalized $\mathrm{sp}^{3}$ sites that were sparsely generated by diazonium reactions in those studies. However, if our dodecyl addends are strongly clustered, then the typical length of pristine SWCNT segments in the fluorescence-quenched nanotubes could be much larger than $5 \mathrm{~nm} .^{20}$

The pronounced dependence on SWCNT diameter found for the defunctionalization activation energy suggests a new strategy for nanotube structural sorting. In this proposed scheme, a mixed sample containing a wide range of $(n, m)$ species would first be individualized and heavily functionalized using reductive alkylation or a comparable method. Then it would undergo pyrolysis at a temperature and duration chosen to selectively defunctionalize SWCNTs with diameters larger than a targeted value. Nanotubes with smaller diameters would retain their addends and could be selectively dissolved with the aid of bath sonication into organic solvents such as chloroform while the defunctionalized ones would not dissolve. Centrifugation would allow physical separation of the two fractions. In the next step of sorting, the smaller diameter (still functionalized) material would be pyrolyzed at a slightly higher temperature and then reprocessed as described above to recover a fraction enriched in diameters having defunctionalization thresholds between the temperatures of the two pyrolysis treatments. Iteration should permit diameter sorting at a useful level of resolution. This scheme could offer some advantages compared to sorting by techniques such as density gradient ultracentrifugation: better scalability, avoidance of surfactants, and less nanotube shortening and damage from ultrasonic processing.

\section{CONCLUSIONS}

Using a combination of spectroscopic and gravimetric measurements, we have investigated how the thermal defunctionalization of alkylated SWCNTs depends on pyrolysis temperature and nanotube structure. The defunctionalization was monitored by mass loss, the disappearance of the Raman D band, 
the reappearance of van Hove features in absorption, and the restoration of characteristic near-IR fluorescence emission. We find that samples can be restored to essentially pristine condition by heating in an inert atmosphere for 30 minutes at temperatures between 150 and $500{ }^{\circ} \mathrm{C}$. Moreover, the threshold temperatures under these conditions are a strong function of SWCNT structure, as larger diameter nanotubes are more easily defunctionalized than smaller diameter species. The activation energy for addend loss is estimated to increase by a factor of $\sim 1.44$ as nanotube diameter decreases from 1.24 to $0.76 \mathrm{~nm}$. This strong variation may enable a novel scalable approach to SWCNT structural sorting based on selective defunctionalization.

\section{MATERIALS AND METHODS}

Dodecyl Functionalization of SWCNTs. Our functionalized samples were prepared according to the method reported by Billups and co-workers for reductive alkylation of SWCNTs using metallic lithium and dodecyl iodide in liquid ammonia. ${ }^{3,5} \mathrm{In}$ a fume hood, a $20 \mathrm{mg}$ sample of raw SWCNTs produced in the Rice University HiPco reactor ${ }^{26,27}$ was added to a flame-dried $100 \mathrm{~mL}$ three neck round bottom flask and approximately $60 \mathrm{~mL}$ of $\mathrm{NH}_{3}$ was condensed into the flask. Granular lithium metal (Sigma-Aldrich, 99\% pure) was then added to the flask followed by the addition of $1.90 \mathrm{~g}$ of 1 iodododecane (Sigma-Aldrich). The reaction mixture was magnetically stirred overnight with slow evaporation of $\mathrm{NH}_{3}$. Next, the flask was cooled in an ice bath as methanol and then water were slowly added. After neutralization with $10 \% \mathrm{HCl}$, the functionalized SWCNT product was extracted into hexanes and washed several times with water. The hexane layer was then filtered through a $0.2 \mu \mathrm{m}$ PTFE membrane and residual products were washed with ethanol and chloroform and dried overnight in a vacuum oven at $80{ }^{\circ} \mathrm{C}$. The resulting product was highly soluble in chloroform. The scheme used for dodecylation is shown in Figure S1. 
Thermal Defunctionalization of Dodecylated SWCNTs. The dodecylated SWCNTs were defunctionalized by continuous heating at specific temperatures under argon. Samples of approximately $2 \mathrm{mg}$ were precisely weighed, wrapped in a small pieces of copper foil, and placed inside a fused silica tube that was evacuated to 6.4 Torr (Figure S2). The tube was inserted into a controlled tube furnace (Lindberg Blue M) and continuously heated for $30 \mathrm{~min}$ at a constant selected temperature in the range of 100 to $500{ }^{\circ} \mathrm{C}$. To avoid oxidation of SWCNTs, a continuous flow of Ar (200 sccm) was maintained during this pyrolysis. Each sample was weighed before and after pyrolysis using a Cahn model C-31 microbalance.

Thermogravimetric Analysis (TGA) of Dodecylated SWCNTs. We performed thermogravimetric analysis of $\mathrm{C}_{12} \mathrm{H}_{25}$-SWCNT samples using a model Q-600 Simultaneous TGA/DSC instrument (TA Instruments). For continuous decomposition experiments (see Fig. 2a), a dried $\sim 2 \mathrm{mg}$ sample was heated from 50 to $500{ }^{\circ} \mathrm{C}$ at a rate of $2{ }^{\circ} \mathrm{C} / \mathrm{min}$ in a flowing argon atmosphere (flow rate 100 sccm).

To obtain stepwise isothermal TGA data on functionalized SWCNTs, samples were isothermally heated under flowing argon for $20 \mathrm{~min}$ at each temperature, with ramps of $5{ }^{\circ} \mathrm{C} / \mathrm{min}$ between temperature plateaus.

Raman Spectroscopy. We measured Raman spectra of solid covalently functionalized SWCNT samples using a Renishaw 1000 micro-Raman spectrometer with a $633 \mathrm{~nm}$ excitation laser source. Spectral signals were acquired from small regions of dried samples placed on silicon substrate, using 10 to $30 \mathrm{~s}$ accumulation times and a $10 \%$ laser power setting. Our reported spectra are baseline-corrected and normalized to the dominant $\mathrm{G}$ band peak near $1590 \mathrm{~cm}^{-1} \cdot{ }^{11}$ Raman spectra of SWCNTs in aqueous suspension were measured with 671 or $740 \mathrm{~nm}$ excitation using model NS3 or NS2 NanoSpectralyzers (Applied NanoFluorescence). 


\section{Dispersion and Characterization of defunctionalized SWCNTs. Following pyrolysis,}

dodecylated SWCNTs samples were ultrasonically dispersed in aqueous surfactant solution for optical characterization. Approximately $0.5 \mathrm{mg}$ of sample was added to a glass vial containing $3.5 \mathrm{~mL}$ of $1 \%$ (w/v) aqueous sodium deoxycholate (Fisher Scientific, 99\% grade) and processed for $1 \mathrm{~h}$ in an ultrasonic bath (Sharpertek, Stamina XP) at room temperature followed by $30 \mathrm{~min}$ of tip sonication (Microson XL with a $3 \mathrm{~mm}$ probe) at an output power of $8 \mathrm{~W}$ with a duty cycle of $45 \mathrm{~s}$ on, $15 \mathrm{~s}$ off. To avoid overheating the sample during tip sonication, the vial was immersed in a water bath. Residual bundles and metallic catalyst in the dispersed samples were removed by centrifugation for 10 min at $10 \mathrm{krpm}$ $(\sim 10,000 \mathrm{x}$ g) in a benchtop centrifuge (Baxter Scientific, Biofuge-13). The resultant supernatants were used for optical measurements. We found that nanotube samples that were pyrolyzed below $200{ }^{\circ} \mathrm{C}$ did not suspend in aqueous surfactant solution.

Characterization of dispersed pyrolyzed samples by absorption and fluorescence spectroscopy was performed with a model NS2 NanoSpectralyzer. We measured Vis-SWIR absorption spectra from 400 to $1400 \mathrm{~nm}$ through a $1 \mathrm{~cm}$ path length relative to a reference cell containing only a $1 \%(\mathrm{w} / \mathrm{v})$ surfactant solution. SWIR fluorescence was captured from 900 to $1400 \mathrm{~nm}$ with sample excitation at $659 \mathrm{~nm}$ (Fig. 7). Spectral resolutions were $1 \mathrm{~nm}$ for visible spectra and $4 \mathrm{~nm}$ in the SWIR region. When needed, we measured full excitation-emission scans using a Spex Fluorolog 3-211 spectrofluorometer (Horiba J-Y) equipped with a liquid nitrogen cooled single-channel InGaAs detector. The excitation wavelength was typically scanned from 500 to $850 \mathrm{~nm}$ in $5 \mathrm{~nm}$ steps and the emission wavelength was scanned from 900 to $1400 \mathrm{~nm}$ in $2 \mathrm{~nm}$ steps. Spectral slit widths were $10 \mathrm{~nm}$ for excitation and $6 \mathrm{~nm}$ for emission. Measured emission signals were corrected to account for wavelength-dependent excitation power and detection system sensitivity. A Schott RG-830 (3 mm thick) filter in the emission channel blocked scattered excitation light. 
Acknowledgments. This research was supported by grants from the National Science Foundation (CHE1409698) and the Welch Foundation (C-0807 to RBW, and C-0490 to WEB). We are grateful to Mr. Xiujun Fan for expert technical assistance with pyrolysis and TGA apparatus.

Supporting Information Available: Additional spectral and AFM data . This material is available free of charge via the Internet at http://pubs.acs.org. 


\section{References}

1. Hirsch, A.; Vostrowsky, O. Functionalization of Carbon Nanotubes. Topics in Current Chemistry 2005, 245, 193-237.

2. Moore, V. C.; Strano, M. S.; Haroz, E. H.; Hauge, R. H.; Smalley, R. E. Individually Suspended Single-Walled Carbon Nanotubes in Various Surfactants. Nano Lett. 2003, 3, 1379-1382.

3. Liang, F.; Sadana, A. K.; Peera, A.; Chattopadhyay, J.; Gu, Z.; Hauge, R. H.; Billups, W. E. A Convenient Route to Functionalized Carbon Nanotubes. Nano Lett. 2004, 4, 1257-1260.

4. Dyke, C. A.; Stewart, M. P.; Maya, F.; Tour, J. M. Diazonium-Based Functionalization of Carbon Nanotubes: XPS and GC-MS Analysis and Mechanistic Implications. Synlett 2004, 155160.

5. Liang, F.; Alemany, L. B.; Beach, J. M.; Billups, W. E. Structure Analyses of Dodecylated Single-Walled Carbon Nanotubes. J. Am. Chem. Soc. 2005, 127, 13941-13948.

6. Chattopadhyay, J.; Sadana, A. K.; Liang, F.; Beach, J. M.; Yunxuan, X.; Hauge, R. H.; Billups, W. E. Carbon Nanotube Salts. Arylation of Single-Wall Carbon Nanotubes. Organic Letters 2005, 7, 4067-4069.

7. Nish, A.; Hwang, J. Y.; Doig, J.; Nicholas, R. J. Highly Selective Dispersion of Singlewalled Carbon Nanotubes Using Aromatic Polymers. Nat. Nanotechnol. 2007, 2, 640-646.

8. Doyle, C. D.; Rocha, J.-D. R.; Weisman, R. B.; Tour, J. M. Structure-Dependent Reactivity of Semiconducting Single-Walled Carbon Nanotubes With Benzenediazonium Salts. J. Am. Chem. Soc. 2008, 130, 6795-6800.

9. Bosch, S.; Zeininger, L.; Hauke, F.; Hirsch, A. A Supramolecular Approach for the Facile Solubilization and Separation of Covalently Functionalized Single-Walled Carbon Nanotubes. 2014, 20, 2537-2541.

10. Gebhardt, B.; Graupner, R.; Hauke, F.; Hirsch, A. A Novel Diameter-Selective Functionalization of SWCNTs With Lithium Alkynylides. 2010, 1494-1501.

11. Graupner, R. Raman Spectroscopy of Covalently Functionalized Single-Wall Carbon Nanotubes. J. Raman Spectrosc. 2007, 38, 673-683.

12. Alemany, L. B.; Zhang, L.; Zeng, L.; Edwards, C. L.; Barron, A. R. Solid-State NMR Analysis of Fluorinated Single-Walled Carbon Nanotubes: Assessing the Extent of Fluorination. Chem. Mater. 2007, 19, 735-744.

13. Dresselhaus, M. S.; Dresselhaus, G.; Saito, R.; Jorio, A. Raman Spectroscopy of Carbon Nanotubes. Physics Reports 2005, 409, 47-99. 
14. Bachilo, S. M.; Strano, M. S.; Kittrell, C.; Hauge, R. H.; Smalley, R. E.; Weisman, R. B. Structure-Assigned Optical Spectra of Single-Walled Carbon Nanotubes. Science 2002, 298, 2361-2366.

15. Weisman, R. B.; Bachilo, S. M. Dependence of Optical Transition Energies on Structure for Single-Walled Carbon Nanotubes in Aqueous Suspension: An Empirical Kataura Plot. Nano Lett. 2003, 3, 1235-1238.

16. Shen, L.; Li, J. Equilibrium Structure and Strain Energy of Single-Walled Carbon Nanotubes. 2005,71 .

17. Kelly, K. F.; Chiang, I. W.; Mickelson, E. T.; Hauge, R. H.; Margrave, J. L.; Wang, X.; Scuseria, G. E.; Radloff, C.; Halas, N. J. Insight into the Mechanism of Sidewall Functionalization of Single-Walled Nanotubes: An STM Study. Chem. Phys. Lett. 1999, 313, 445-450.

18. Worsley, K. A.; Moonoosawmy, K. R.; Kruse, P. Long-Range Periodicity in Carbon Nanotube Sidewall Functionalization. Nano Lett. 2004, 4, 1541-1546.

19. Van Lier, G.; Ewels, C. P.; Zuliani, F.; De Vita, A.; Charlier, J. C. Theoretical Analysis of Fluorine Addition to Single-Walled Carbon Nanotubes: Functionalization Routes and Addition Patterns. J. Phys. Chem. B 2005, 109, 6153-6158.

20. Deng, S.; Zhang, Y.; Brozena, A. H.; Mayes, M. L.; Banerjee, P.; Chiou, W. A.; Rubloff, G. W.; Schatz, G. C.; Wang, Y. Confined Propagation of Covalent Chemical Reactions on SingleWalled Carbon Nanotubes. 2011, 2.

21. Strano, M. S.; Dyke, C. A.; Usrey, M. L.; Barone, P. W.; Allen, M. J.; Shan, H.; Kittrell, C.; Hauge, R. H.; Tour, J. M.; Smalley, R. E. Electronic Structure Control of Single-Walled Carbon Nanotube Functionalization. Science 2003, 301, 1519-1522.

22. Piao, Y.; Meany, B.; Powell, L. R.; Valley, N.; Kwon, H.; Schatz, G. C.; Wang, Y. Brightening of Carbon Nanotube Photoluminescence Through the Incorporation of Sp3 Defects. Nature Chemistry 2013, 5, 840-845.

23. Naumov, A. V.; Ghosh, S.; Tsyboulski, D. A.; Bachilo, S. M.; Weisman, R. B. Analyzing Absorption Backgrounds in Single-Walled Carbon Nanotube Spectra. ACS Nano 2011, 5, 16391648.

24. Cognet, L.; Tsyboulski, D.; Rocha, J.-D. R.; Doyle, C. D.; Tour, J. M.; Weisman, R. B. Stepwise Quenching of Exciton Fluorescence in Carbon Nanotubes by Single-Molecule Reactions. Science 2007, 316, 1465-1468.

25. Siitonen, A. J.; Tsyboulski, D. A.; Bachilo, S. M.; Weisman, R. B. Surfactant-Dependent Exciton Mobility in Single-Walled Carbon Nanotubes Studied by Single-Molecule Reactions. Nano Lett. 2010, 10, 1595-1599. 
26. Bronikowski, M. J.; Willis, P. A.; Colbert, D. T.; Smith, K. A.; Smalley, R. E. Gas-Phase Production of Carbon Single-Walled Nanotubes From Carbon Monoxide via the HiPco Process: A Parametric Study. J. Vac. Sci. Technol. 2001, A19, 1800-1805.

27. Nikolaev, P.; Bronikowski, M. J.; Bradley, R. K.; Rohmund, F.; Colbert, D. T.; Smith, K. A.; Smalley, R. E. Gas-Phase Catalytic Growth of Single-Walled Carbon Nanotubes From Carbon Monoxide. Chem. Phys. Lett. 1999, 313, 91-97. 
Table 1. Structure-Dependent Defunctionalization Threshold Results, as deduced from

Raman Data. Threshold temperatures have estimated uncertainties of $\pm 10{ }^{\circ} \mathrm{C}$.

$\begin{array}{cccccc}(n, m) & \begin{array}{c}\text { Raman excitation } \\ \text { wavelength }(\mathrm{nm})\end{array} & \text { diameter }(\mathrm{nm}) & \begin{array}{c}\text { roll-up } \\ \text { angle }\left(^{\circ}\right)\end{array} & \begin{array}{c}\text { threshold } \\ \text { temperature }\left({ }^{\circ} \mathrm{C}\right)\end{array} & \begin{array}{c}\text { relative activation } \\ \text { energy }\end{array} \\ (6,5) & 785 & 0.757 & 27.0 & 320 & 1.44 \\ (8,3) & 671 & 0.782 & 15.3 & 300 & 1.39 \\ (7,5) & 633 & 0.829 & 24.5 & 240 & 1.24 \\ (10,2) & 740 & 0.884 & 9.0 & 220 & 1.19 \\ (7,6) & 633 & 0.895 & 27.5 & 210 & 1.17 \\ (10,3) & 633 & 0.936 & 12.7 & 200 & 1.15 \\ (11,3) & 785 & 1.014 & 11.7 & 190 & 1.12 \\ (12,2) & 671 & 1.041 & 7.6 & 180 & 1.12 \\ (10,5) & 785 & 1.050 & 19.1 & 180 & 1.10 \\ (12,3) & 633 & 1.08 & 10.9 & 180 & 1.10 \\ (9,7) & 785 & 1.103 & 25.9 & 160 & 1.05 \\ (9,8) & 785 & 1.170 & 28.1 & 160 & 1.05 \\ (9,9) & 633 & 1.238 & 30.0 & 140 & 1\end{array}$


Table 2. Gravimetric and Raman Data for Pyrolysis of Dodecylated SWCNTs

\begin{tabular}{|c|c|c|c|c|}
\hline $\begin{array}{c}\text { pyrolysis } \\
\text { temperature }\left({ }^{\circ} \mathrm{C}\right)\end{array}$ & $\begin{array}{c}\text { weight loss } \\
(\%)\end{array}$ & $\begin{array}{l}\text { Raman }\left(\mathrm{I}_{\mathrm{D}} / \mathrm{I}_{\mathrm{G}}\right) \\
\text { peak ratio }\end{array}$ & $\begin{array}{l}\mathrm{C}_{12} \mathrm{H}_{25} / \mathrm{C} \\
\text { ratio }\end{array}$ & $\begin{array}{l}\mathrm{C} / \mathrm{C}_{12} \mathrm{H}_{25} \\
\text { ratio }\end{array}$ \\
\hline 25 & 0.00 & 1.08 & 0.089 & 11.2 \\
\hline 100 & 3.65 & 1.06 & 0.084 & 11.9 \\
\hline 120 & 6.32 & 1.04 & 0.080 & 12.5 \\
\hline 140 & 9.58 & 1.01 & 0.075 & 13.3 \\
\hline 160 & 15.65 & 0.83 & 0.066 & 15.1 \\
\hline 180 & 20.43 & 0.62 & 0.059 & 17.0 \\
\hline 200 & 29.71 & 0.40 & 0.0444 & 22.5 \\
\hline 220 & 31.15 & 0.31 & 0.0421 & 23.7 \\
\hline 240 & 35.25 & 0.29 & 0.0354 & 28.2 \\
\hline 260 & 38.54 & 0.25 & 0.0299 & 33.4 \\
\hline 280 & 39.06 & 0.21 & 0.0291 & 34.4 \\
\hline 300 & 43.83 & 0.17 & 0.0210 & 47.7 \\
\hline 320 & 44.74 & 0.16 & 0.0194 & 51.5 \\
\hline 340 & 46.81 & 0.15 & 0.0158 & 63.3 \\
\hline 360 & 46.25 & 0.14 & 0.0168 & 59.6 \\
\hline 380 & 49.45 & 0.13 & 0.0112 & 89.6 \\
\hline 400 & 51.57 & 0.13 & 0.0074 & 135 \\
\hline 420 & 52.12 & 0.11 & 0.0064 & 156 \\
\hline 440 & 52.89 & 0.10 & 0.0050 & 199 \\
\hline 460 & 53.71 & 0.09 & 0.0036 & 281 \\
\hline 480 & 55.11 & 0.07 & 0.0010 & 986 \\
\hline 500 & 55.45 & 0.06 & 0.0004 & 2460 \\
\hline
\end{tabular}


a.

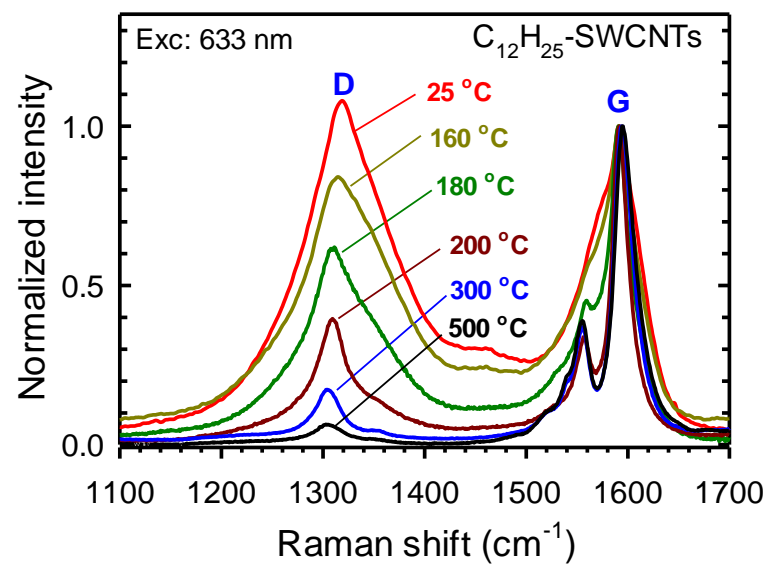

c.

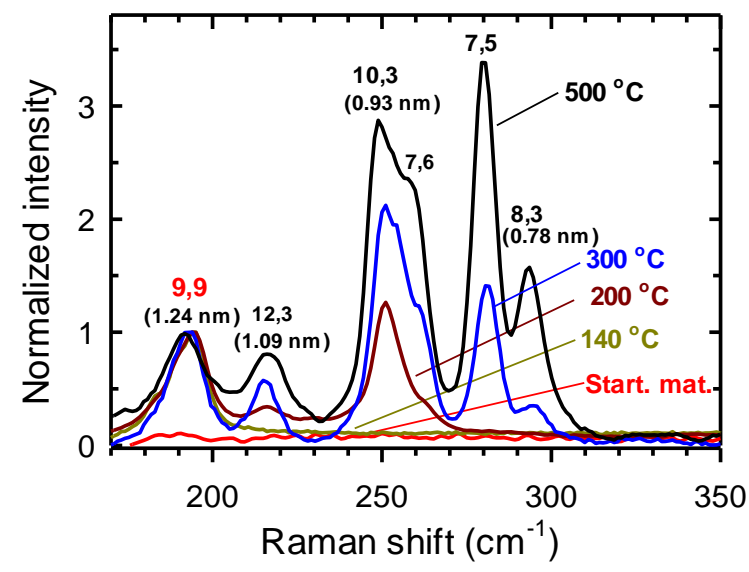

b.

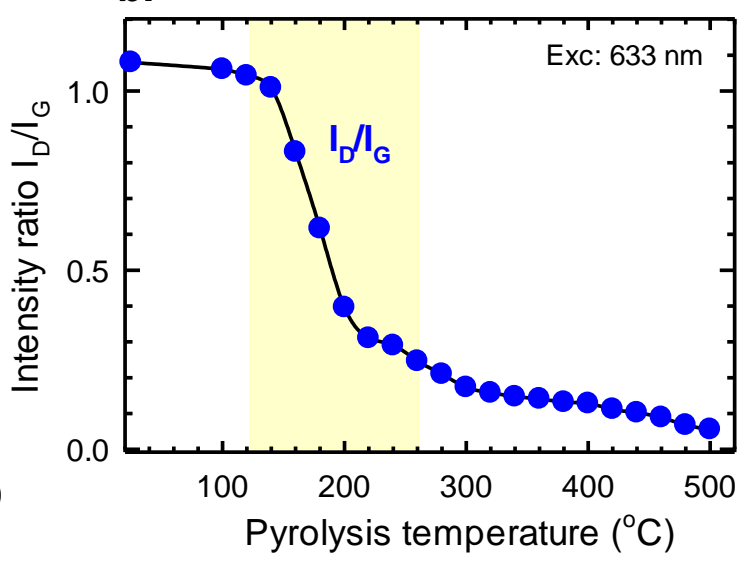

d.

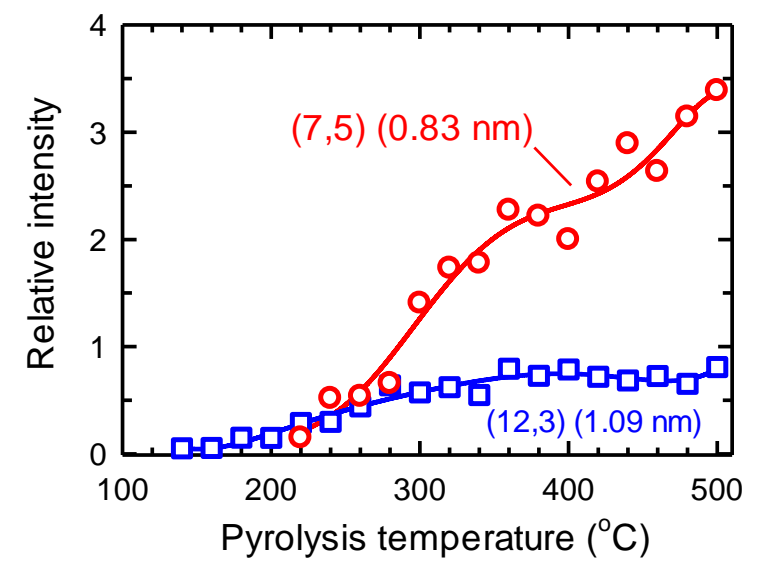

Figure 1. Raman characterization of solid samples of $\mathrm{C}_{12} \mathrm{H}_{25}$-functionalized SWCNTs following pyrolysis at different temperatures. (a) Raman spectra normalized to $G$ peak, showing temperature dependent changes in disorder (D) band. (b) D / G peak intensity ratio vs. pyrolysis temperature for the data in frame (a). (c) Spectra of radial breathing modes (RBMs), normalized to the peak near $\sim 193 \mathrm{~cm}^{-1}$, for a set of samples pyrolyzed at different temperatures and for the unpyrolyzed starting material. (d) RBM peak intensity (relative to peak near $\sim 193 \mathrm{~cm}^{-1}$ ) vs. pyrolysis temperature for the $(7,5)$ and $(12,3)$ species. 

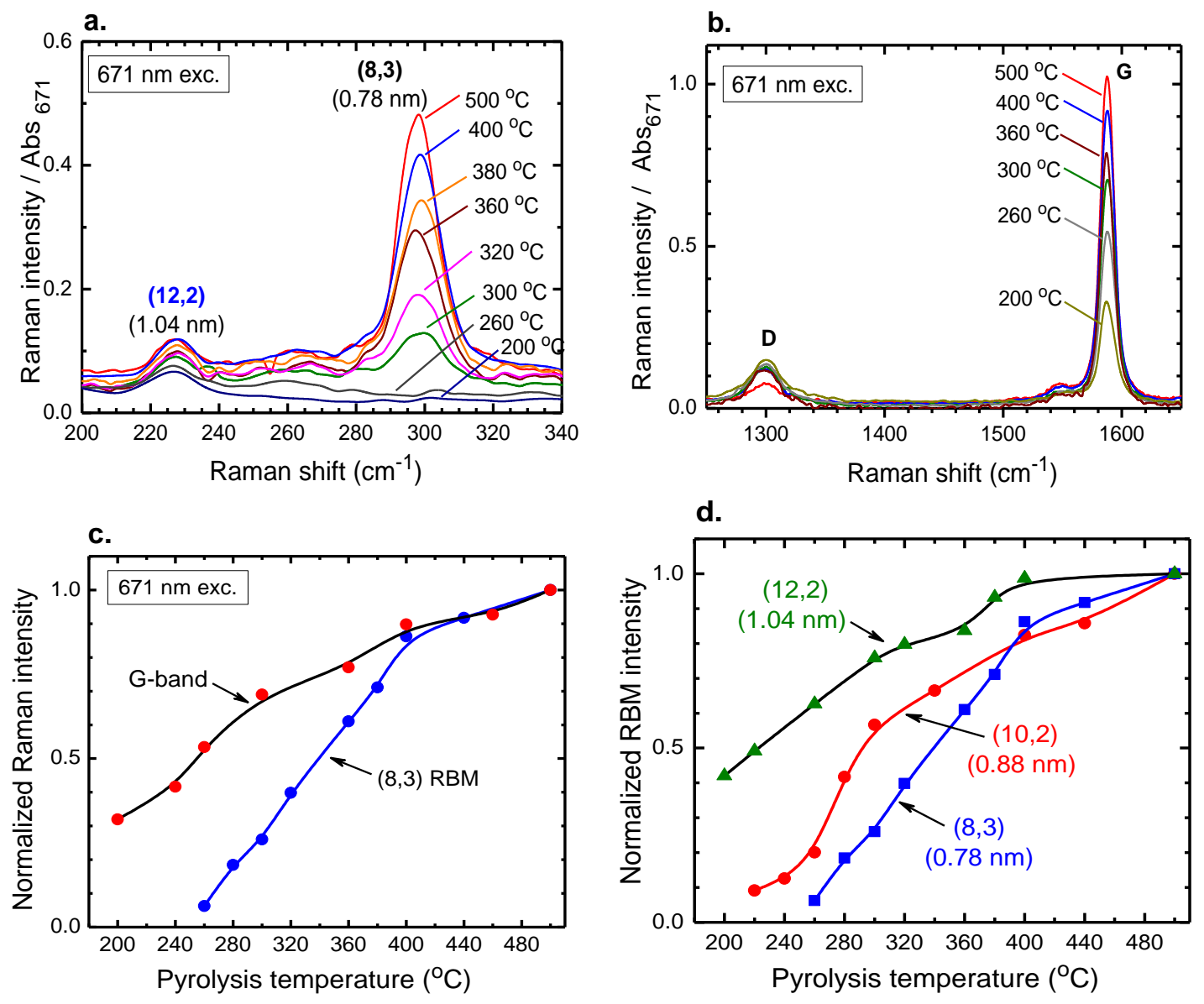

Figure 2. Resonant Raman spectral data on surfactant-coated liquid suspensions of dodecylated SWCNTs that had been pyrolyzed at different temperatures. All samples were adjusted to an absorbance of 0.2 at the $671 \mathrm{~nm}$ excitation wavelength. (a) RBM region spectra following pyrolysis at different temperatures. (b) Spectra in the D- and G-band regions. (c) Peak intensities (normalized to $500{ }^{\circ} \mathrm{C}$ values) of the G-band and $(8,3)$ RBM band $v s$. pyrolysis temperature. (d) Peak RBM intensities (normalized to $500{ }^{\circ} \mathrm{C}$ values) vs. pyrolysis temperature for the $(8,3),(10,2)$, and $(12,2)$ species. The $(10,2)$ data were obtained using a $740 \mathrm{~nm}$ excitation wavelength. 

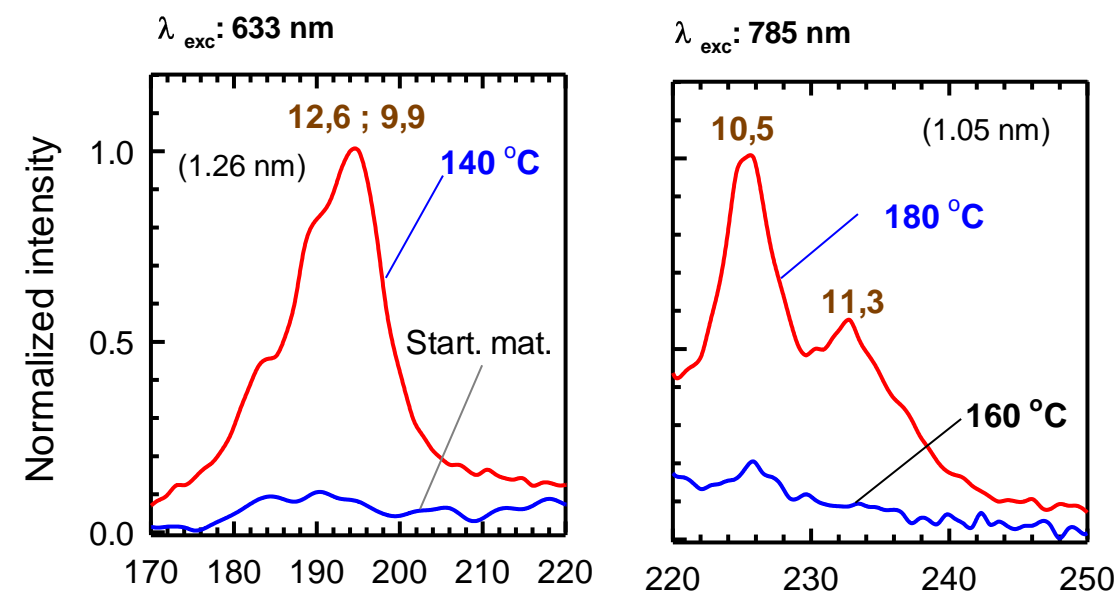

$\lambda_{\text {exc }}: 633 \mathrm{~nm}$
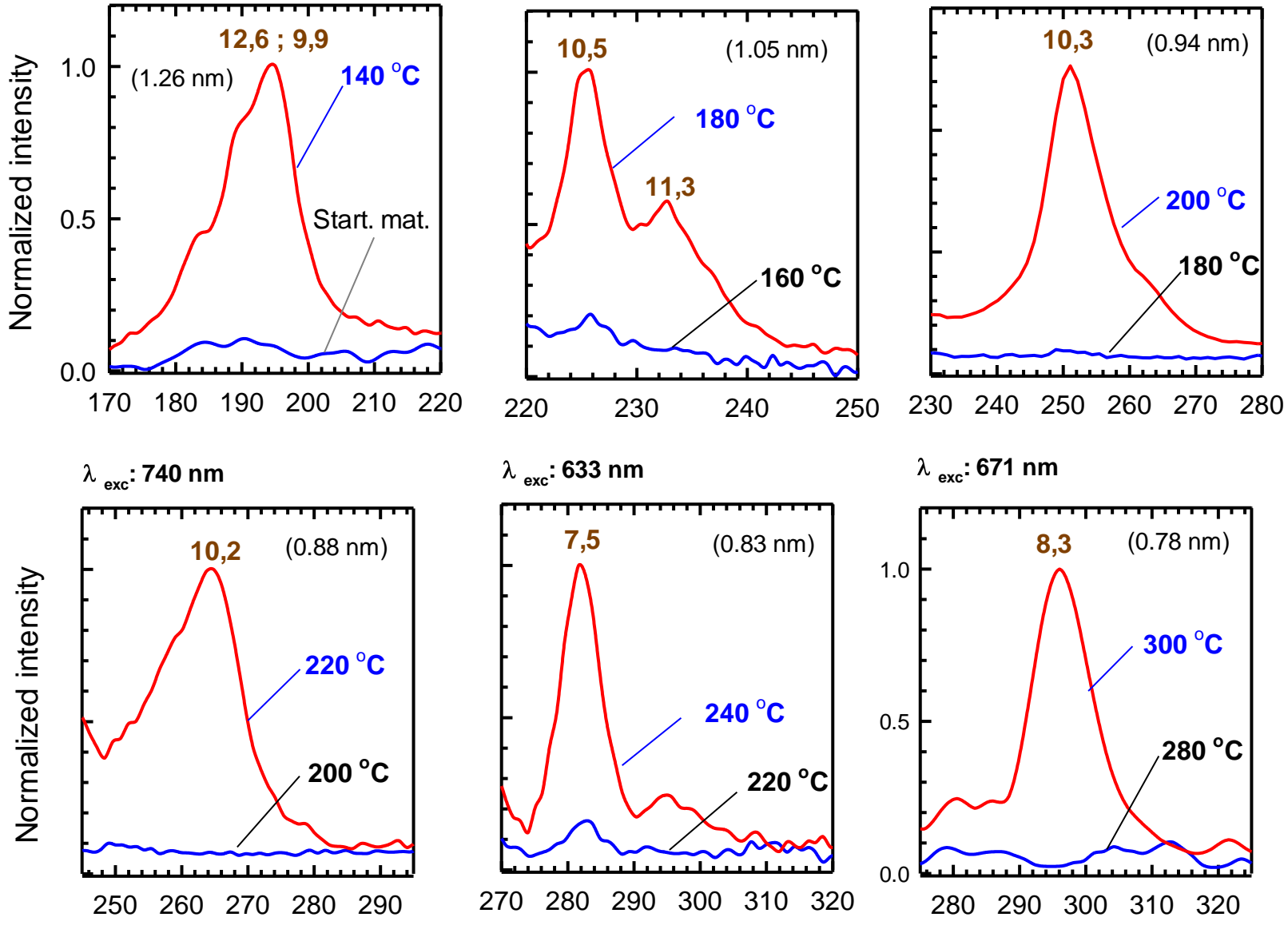

$\lambda_{\text {exc }}: 633 \mathrm{~nm}$

$\lambda_{\text {exc }}: 671 \mathrm{~nm}$
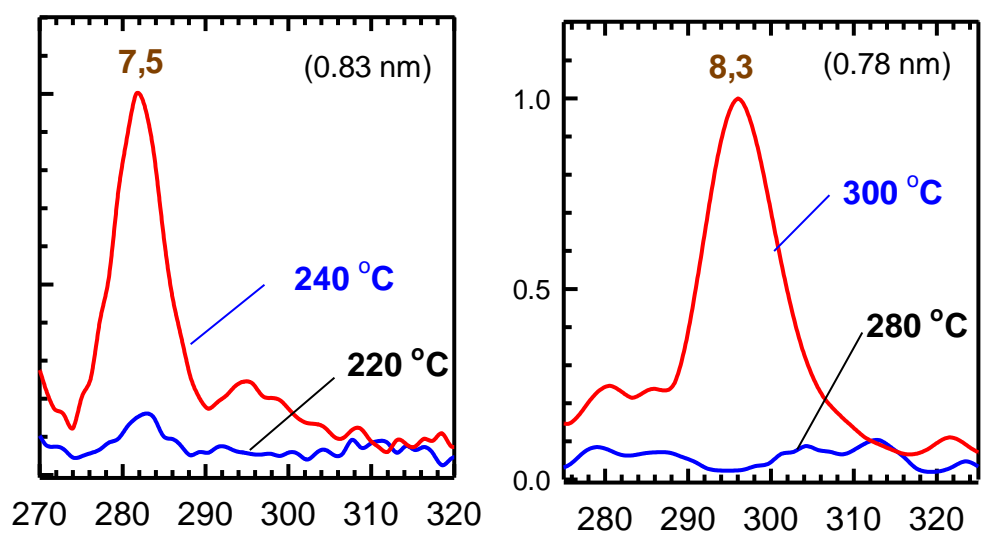

Raman shift $\left(\mathrm{cm}^{-1}\right)$

Figure 3. Raman RBM spectra illustrating the varying onsets of defunctionalization for different $(n, m)$ species. In each frame the pair of traces is shown on the same intensity scale and represent samples that differ only in their pyrolysis temperatures, as labeled. 


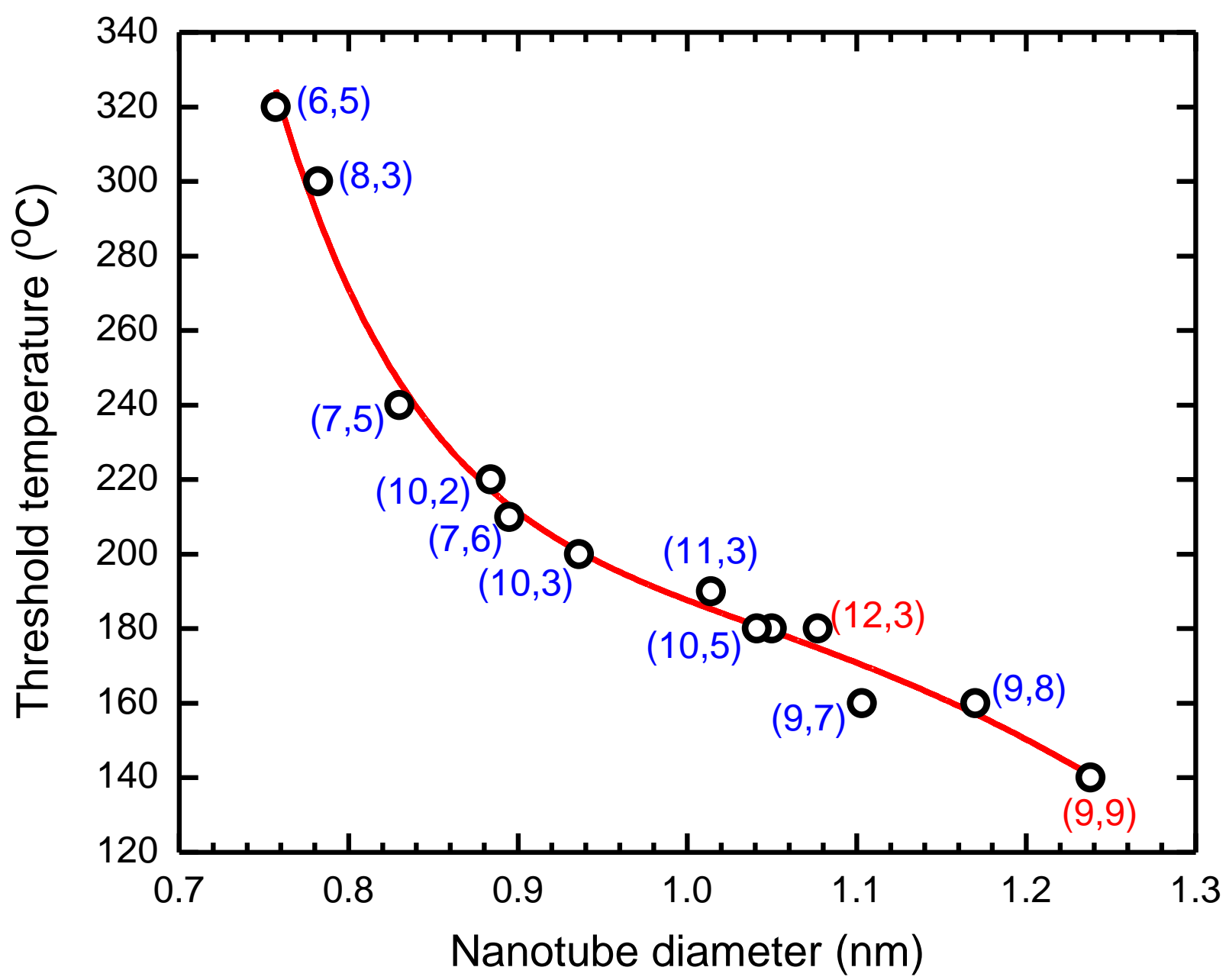

Figure 4. Defunctionalization threshold temperatures for 30 minute pyrolysis of dodecylated SWCNTs plotted vs. nanotube diameter. Data points were obtained from Raman RBM spectral measurements and are labeled with the corresponding $(n, m)$ values in blue text for semiconducting species and in red for metallic. The smooth curve is a guide for the eye. 
a.

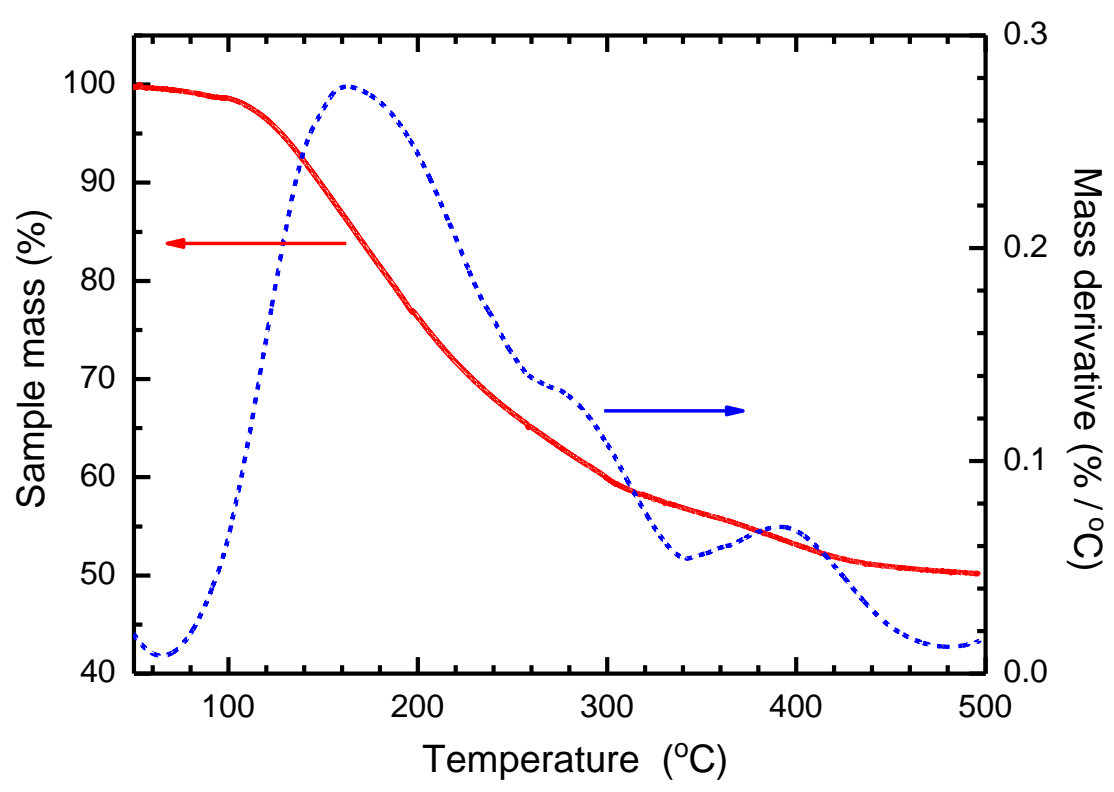

b.

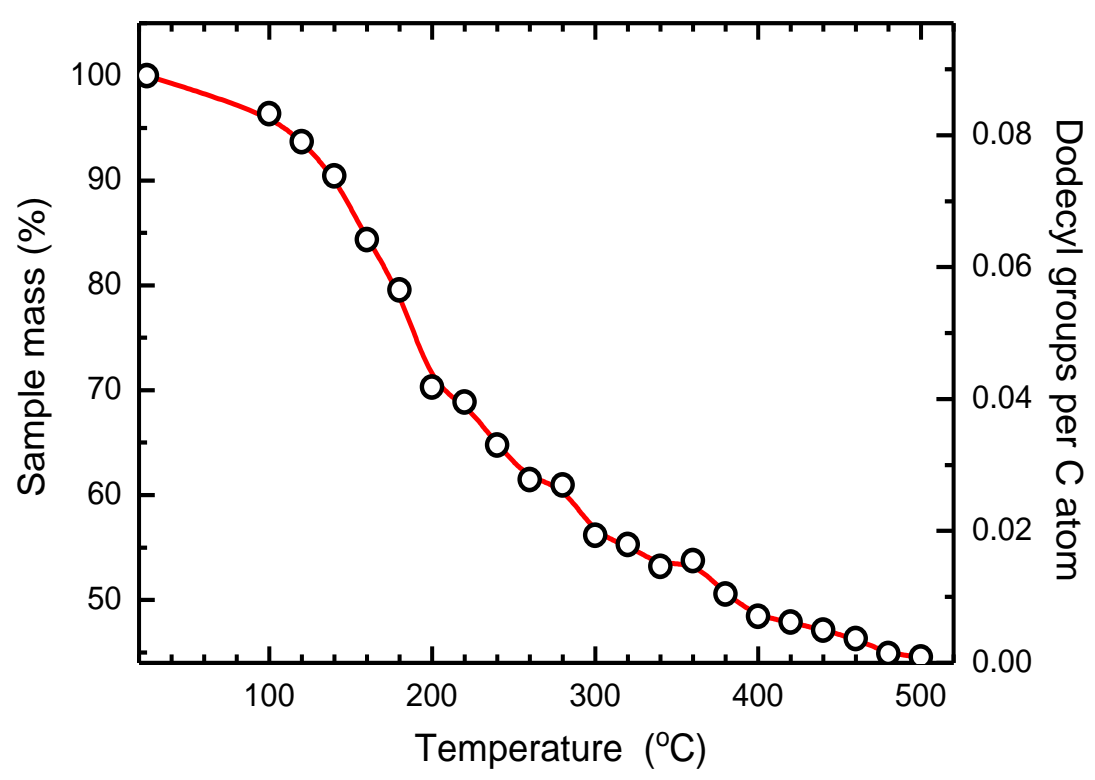

Figure 5. (a) TGA data showing relative mass versus temperature of a single dodecylated SWCNT sample during heating in argon at a rate of $2{ }^{\circ} \mathrm{C} \mathrm{min}^{-1}$ (red solid curve, left scale), and the corresponding mass loss derivative (blue dotted curve, right scale). (b) Relative samples masses remaining after 30 minute pyrolysis treatments at different fixed temperatures for 22 replicate samples of docedylated SWCNTs. The left scale shows final percentage of initial sample mass and the right scale shows the corresponding final ratios of $\mathrm{C}_{12} \mathrm{H}_{25}$ addends per nanotube carbon atom. 


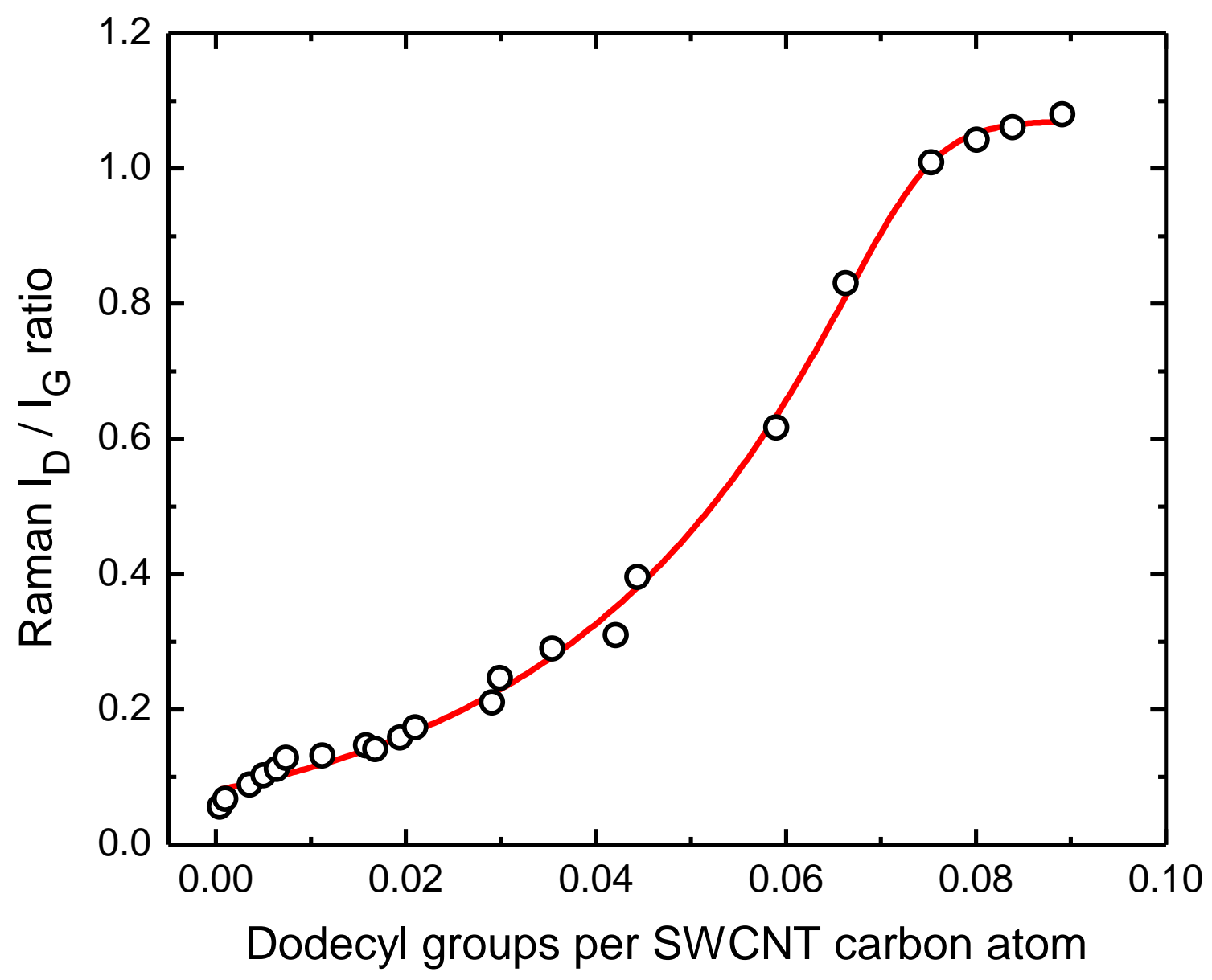

Figure 6. Deduced relation between Raman D / G band peak intensity ratio and the average number of dodecyl groups per nanotube carbon atom. Analysis was based on data from TGA (Figure 5) and 633 nm Raman spectra (Figure 1). 
a.

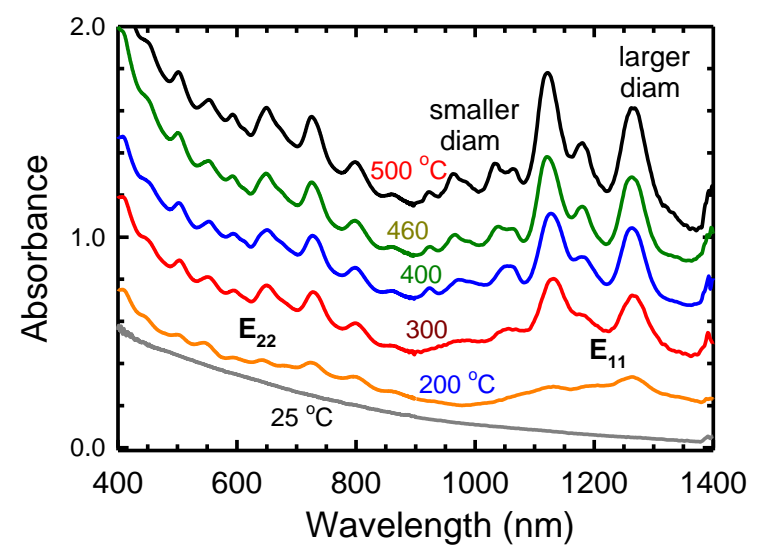

C.

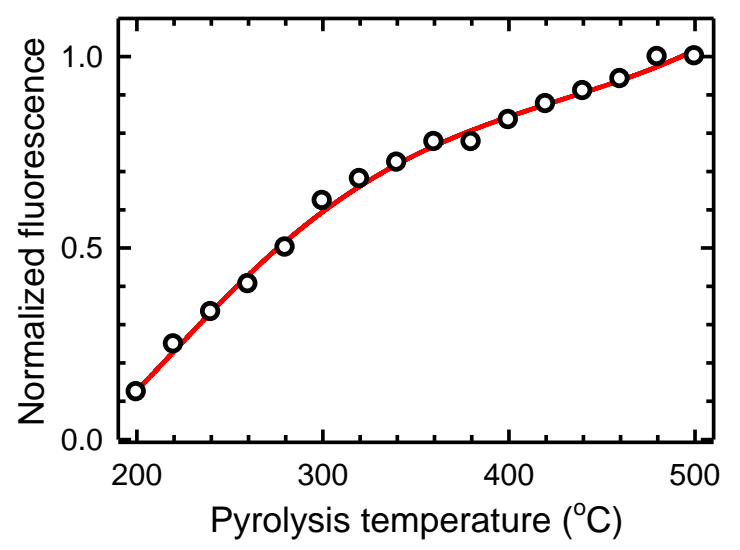

b.

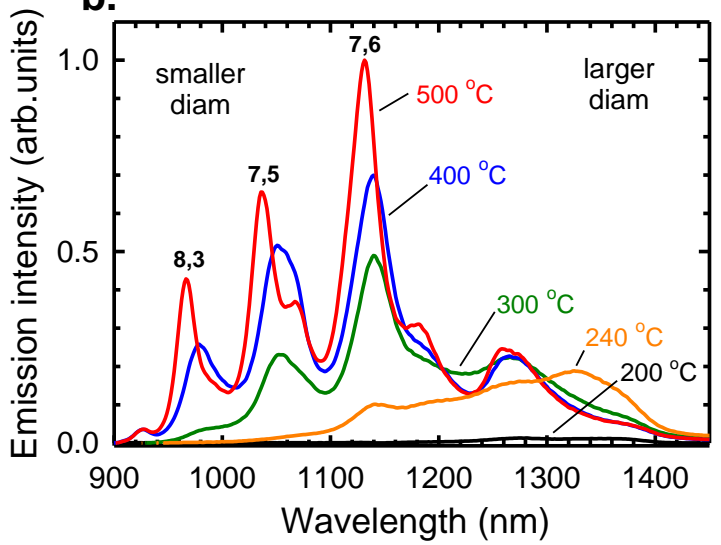

d.

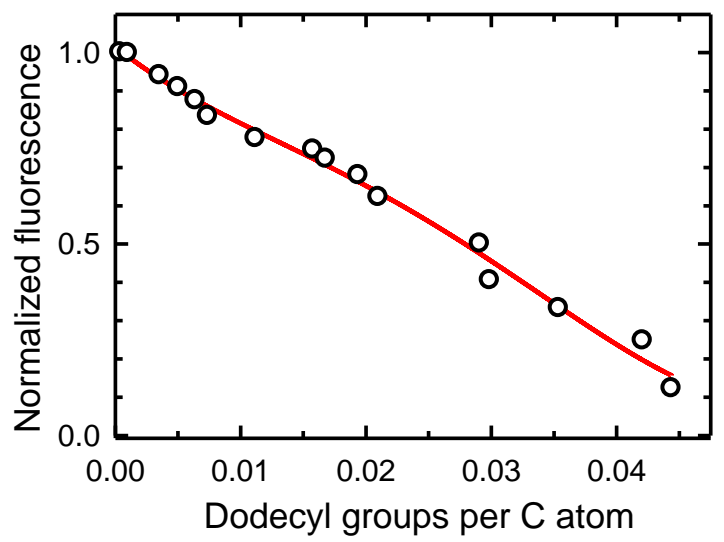

Figure 7. Optical spectra of pyrolyzed dodecylated SWCNT samples in aqueous surfactant suspension. (a) Absorption spectra before pyrolysis (gray trace labeled $25^{\circ} \mathrm{C}$ ) and after 30 minutes of pyrolysis at the labeled temperatures between 200 and $500{ }^{\circ} \mathrm{C}$. Spectra have been vertically offset for clarity. (b) Fluorescence spectra (excited at $659 \mathrm{~nm}$ ) from the same set of samples. No emission was detected from the unpyrolyzed sample. Prominent emissive peaks are labeled with corresponding $(n, m)$ indices. (c) Spectrally integrated fluorescence intensity $v s$. pyrolysis temperature for samples having equal optical absorbance ( 0.3) at the $659 \mathrm{~nm}$ excitation wavelength. (d) Spectrally integrated fluorescence intensity $v s$. the deduced ratio of dodecyl groups to nanotube carbon atoms. Values are normalized to the intensity after full pyrolysis at $500{ }^{\circ} \mathrm{C}$. 


\section{TOC Figure}
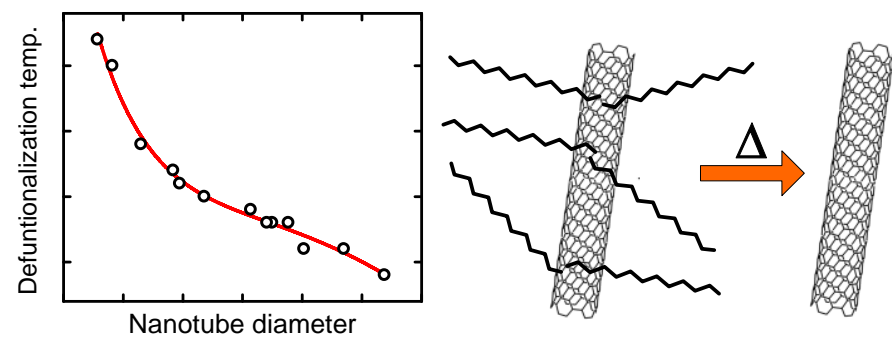